\title{
Emergent Premedication for Contrast Allergy Prior to Endovascular Treatment of Acute Ischemic Stroke
}

\author{
(DD.A. Tonetti, (D) S.M. Desai, (D)A. Morrison, (DB.A. Gross, (D).G. Jovin, (D)B.T. Jankowitz, and (D) A.P. Jadhav
}

\begin{abstract}
BACKGROUND AND PURPOSE: Management of contrast media allergies may lead to treatment delays in patients with acute ischemic stroke undergoing endovascular therapy. The optimal premedication strategy remains unclear. The aim of this report was to analyze our experience with emergent administration of premedication regimens before endovascular therapy.
\end{abstract}

MATERIALS AND METHODS: We retrospectively reviewed prospective data for all patients undergoing endovascular therapy from 2012 to 2019 at an academic comprehensive stroke center. Records of patients with documented contrast allergy were reviewed and analyzed. Data collected included stroke risk factors and characteristics, historical contrast reaction details, premedication regimens administered, and signs or symptoms of allergic reaction developing post-endovascular therapy. Hospital arrival time to endovascular therapy was compared with that in those who did not have a history of contrast allergy.

RESULTS: We analyzed 1521 patients undergoing endovascular therapy; 60 (4\%) had documented contrast allergies and constituted the study cohort. The median age was 73 years (interquartile range, 66-81 years), and 65\% were women. The median time from premedication to contrast was 24 minutes (interquartile range, 0-36 minutes). Forty-three patients (72\%) proceeded directly to endovascular therapy; in 17 patients, the first contrast exposure was CTA. Time from hospital arrival to endovascular therapy was not slower for patients with documented allergies (96 versus 134 minutes, $P=.32$ ). No patients experienced a contrast media reaction.

CONCLUSIONS: In a single-institution cohort study of 60 consecutive patients with documented contrast allergies undergoing endovascular therapy with emergent premedication en route to (or in) the neuroangiography suite, no patients experienced allergic symptoms. This pragmatic approach may be safe for patients who have documented contrast media allergies.

ABBREVIATIONS: EVT = endovascular therapy; ICM = iodinated contrast media; IQR = interquartile range; LVO = large-vessel occlusion

A llergic reactions occur in $0.1 \%-0.6 \%$ of patients after exposure to modern nonionic iodinated contrast media (ICM), of which $1 \%-5 \%$ are severe. ${ }^{1-4}$ Similarly, documentation of contrast dye allergies is not uncommon in electronic health records. ${ }^{3}$ Documentation of a contrast media reaction may lead to delays in care for patients with acute ischemic stroke who require the administration of contrast for cerebrovascular imaging in the form of CTA and/or endovascular therapy $(\mathrm{EVT})$ in an emergent manner.

Received May 6, 2020; accepted after revision June 15.

From the Department of Neurological Surgery (D.A.T., B.A.G.) and Stroke Institute (D.A.T., S.M.D., B.A.G., A.P.J.), University of Pittsburgh Medical Center, Pittsburgh, Pennsylvania; University of Pittsburgh School of Medicine (A.M.), Pittsburgh, Pennsylvania; and Cooper University Hospitals (T.G.J., B.T.J.), Camden, New Jersey.

Please address correspondence to Ashutosh P. Jadhav, MD, Stroke Institute, University of Pittsburgh, Suite C-400, UPMC Presbyterian, 200 Lothrop St, Pittsburgh, PA 15213; e-mail: jadhav.library@gmail.com; @DanTonettiMD; @AshuPJadhav

http://dx.doi.org/10.3174/ajnr.A6720
There are no guidelines regarding antireaction medication paradigms for patients with a documented contrast dye allergy undergoing emergent EVT for large-vessel occlusion (LVO), and practices vary widely. Furthermore, contrast allergy is typically considered a contraindication for enrollment in EVT clinical trials, so high-quality data regarding best practices in this population are lacking. A documented contrast allergy may result in delay and/or avoidance of performing contrast-based cerebrovascular imaging (eg, CTA) per local institutional practices. At our center, a protocol was introduced in which patients presenting with emergent LVO bypass CTA and proceed directly to the neurointerventional suite. These patients were then administered prophylactic premedication as soon as possible for their contrast allergy, with the aim of avoiding delays to revascularization.

The objective of this report was to analyze our institutional experience administering emergent premedication regimens to consecutive patients with a documented history of ICM allergy at the time of emergent EVT. 


\section{MATERIALS AND METHODS}

We performed a retrospective review of prospectively collected patient records at a single academic comprehensive stroke center (University of Pittsburgh Medical Center) from 2012 to 2019. All patients who were referred for EVT for emergent LVO during this time were included if they had a documented history of a reaction to contrast media. Patients were referred for EVT by a fellowship-trained vascular neurologist, and EVT was performed by 1 of 4 neurointerventionalists. This study was approved by our local institutional review board.

The study protocol began when a patient with a documented history of ICM allergy was referred for EVT; patients in the protocol who presented directly to the comprehensive stroke center would bypass noninvasive cerebrovascular imaging (eg, CTA) and proceed directly to the neurointerventional angiography suite. For patients who were transferred from outside facilities, CTA may have been performed per local institutional protocols, and these patients were included in the analysis. Contrast premedication was administered at the discretion of the treating physicians before EVT in all cases. Premedication regimens and dosages were tabulated and recorded, along with the time from premedication administration to contrast administration.

EVT was performed with the patient under monitored anesthesia care or general anesthesia using Isovue-300 (Bracco) and conventional thrombectomy methods. Contrast dosages used for EVT were recorded. The dose of contrast used for the DSA was calculated by subtracting the contrast remaining at the end of the case from the amount of contrast in bottles opened. All patients were monitored by both an anesthesiology team and a trained neurointerventional nurse before, during, and after the procedure. Patients were then observed in a dedicated neurointensive care unit and monitored for symptoms of allergic reaction for 24 hours postprocedure.

Additional data collected and recorded included stroke risk factors and characteristics, historical contrast reaction details (which contrast agent and what type of reaction, when available), the necessity for further treatment of contrast reactions (additional medication, intubation for airway protection), and symptoms of allergic reactions during or after the procedure for up to 24 hours.

\section{Outcome Measures}

The primary outcome was any indication of allergic reaction to contrast media at any point up to 24 hours postprocedure. Symptoms including nausea, emesis, rash, shortness of breath, anaphylaxis, or unexplained hypotension were reviewed and recorded. The need for additional antiallergy medication or intubation was recorded. Time metrics were then compared with those of patients undergoing EVT without documented contrast allergy during the same study period to assess differences or delays in care.

\section{RESULTS}

Between 2012 and 2019, in the setting of an LVO, 1521 patients with acute ischemic stroke underwent EVT. Of those, 60 patients (4\%) had a documented contrast allergy (Figure). Forty-three patients $(43 / 60,72 \%)$ bypassed noninvasive vascular imaging and proceeded directly to the angiography suite; another 17 patients
(28\%) underwent CTA before EVT and were premedicated before CTA. In patients who bypassed CTA, the presence of an LVO was suspected on the basis of CT of the head without contrast findings (ie, hyperdense thrombus sign) or by clinical examination (NIHSS score $>12$ ). Occlusion location was the middle cerebral artery (segment $1, \mathrm{M} 1$ ) in $45 \%$ or the ICA terminus in $27 \%$ (Table 1). The median time from last-known-well to hospital arrival was 4.3 hours (interquartile range $[\mathrm{IQR}]=2.4-8.1$ hours), the median NIHSS score was 18 (IQR $=13-23)$, and the median ASPECTS was 9 (IQR $=8-10)$. Intravenous tPA was administered in 16 patients $(16 / 60,27 \%)$.

Previously documented allergic reactions were tabulated and recorded when available at the time of EVT. Reaction types were as follows: rash $(20 / 60,33 \%)$, anaphylaxis $(10 / 60,17 \%)$, shortness of breath $(5 / 60,8 \%)$, and nausea/emesis $(9 / 60,15 \%)$ with previous ICM administration. Twenty-one patients (35\%) with documented ICM allergy lacked specific details regarding a prior allergic response. At the time of admission, 1 patient (1.7\%) was taking $3 \mathrm{mg}$ of prednisone daily for polymyalgia rheumatica; no other patients were on pre-existing chronic oral steroid regimens.

\section{Premedication Regimen}

Fifty-six patients (93\%) received a combination of corticosteroid and diphenhydramine as premedication immediately before EVT (Table 2). All medications were administered as intravenous pushes. The remaining 4 patients received corticosteroid only. Hydrocortisone was the most commonly used corticosteroid, and the most common dose was $200 \mathrm{mg}$. When methylprednisolone was used ( $n=11,18 \%)$, doses between 100 and $200 \mathrm{mg}$ were used. The average dose of diphenhydramine was $50 \mathrm{mg}$. Ten milligrams of dexamethasone was used in conjunction with diphenhydramine in 1 patient (1.7\%). No patients had adverse effects attributable to corticosteroid or antihistamine administration.

EVT was performed with $88 \%$ of patients achieving modified TICI $2 \mathrm{~b}$ or higher reperfusion using an average contrast dose of $151 \pm 32 \mathrm{~mL}$ of Isovue 300 . The median time from premedication administration to contrast exposure was 24 minutes (IQR = 5-54 minutes) and was not significantly different between those who underwent CTA first versus those who underwent DSA only $(P=.53)$.

\section{Primary Outcome}

No patients experienced intraprocedural or immediate post-EVT reactions manifesting as nausea/emesis, shortness of breath or wheezing, new rash, or anaphylaxis in response to ICM administration. One patient with a known drug reaction with eosinophilia and systemic symptoms syndrome who had an LVO while undergoing an inpatient work-up for a severe cutaneous rash over her flank and back had a persistent rash that did not worsen with EVT. No patients experienced delayed hypersensitivity reactions manifesting as delayed urticaria or shortness of breath within 24 hours post-EVT.

\section{Impact of Premedication Administration on Time}

For patients with documented contrast allergies, the median time from hospital arrival to EVT was 45 minutes (IQR $=23-$ 


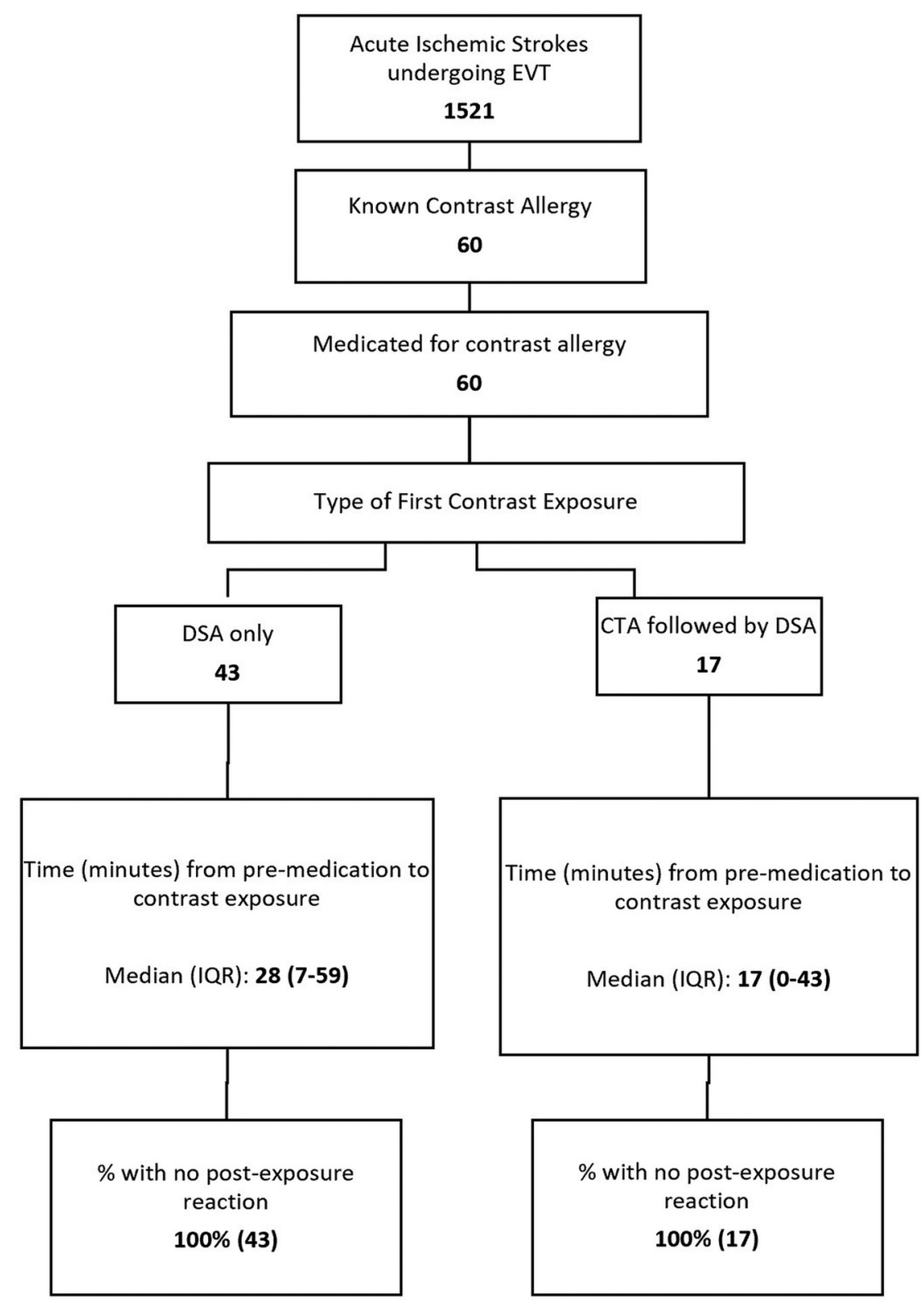

FIGURE. Patient flow diagram.

85 minutes) (Table 3). For all other patients undergoing EVT during the study period, the median time from hospital arrival to puncture was 60 minutes (IQR $=20-105$ minutes), a difference that was not statistically significant $(P=.31)$.

\section{DISCUSSION}

The most important finding of this study is that patients with contrast allergy with LVO who receive emergency pretreatment can safely undergo EVT without delay. Here, we describe a single-institution consecutive series of patients with documented allergies to ICM who were referred for emergent EVT with premedication administered either en route to or in the angiography suite. Among 1521 patients undergoing thrombectomy during the study period, 60 (4\%) had documented contrast allergies. We found that in the cohort of 60 patients referred for EVT with documented ICM allergies during 8 years, undergoing EVT after receiving premedication resulted in no observable reactions.

Contrast dye allergy documentation in the electronic health records is not uncommon; in a cross-sectional analysis of 2.7 million patients in a large health care system in the United States, $1.3 \%$ had documented contrast allergies. ${ }^{3}$ A prior allergic-like reaction to ICM is the most substantial risk factor for a recurrent allergic-like adverse event, ${ }^{5-7}$ and the risk of anaphylactoid reactions is higher in women. ${ }^{8}$ In the setting of premedication, repeat reactions to contrast agents occur uncommonly. ${ }^{9,10}$ When they do occur, reactions are usually of similar severity to the index event and are rarely severe. ${ }^{7,9,10}$ Unfortunately, it is exceptionally common for electronic health records to be ambiguous and lack details regarding imaging-technique-specific allergies or allergies to specific contrast agents. ${ }^{3}$ Regardless of accuracy or reliability, a documented allergy can result in delays in obtaining necessary 
Table 1: Baseline characteristics

\begin{tabular}{lc}
\hline \multicolumn{1}{c}{ Characteristics } & No. (\%) \\
\hline Age (median) (IQR) (yr) & $73(66-81)$ \\
Male sex & $21(35 \%)$ \\
NIHSS score (median) (IQR) & $18(13-23)$ \\
Comorbidities & \\
Hypertension & $50(83 \%)$ \\
Hyperlipidemia & $39(65 \%)$ \\
Atrial fibrillation & $35(58 \%)$ \\
Diabetes mellitus & $19(32 \%)$ \\
ASPECTS (median) (IQR) & $9(8-10)$ \\
TLKW to arrival (median) (IQR) (hr) & $4.3(2.4-8.1)$ \\
IV tPA & $16(27 \%)$ \\
Occlusion location & \\
ICA & $16(27 \%)$ \\
MCA-M1 & $27(45 \%)$ \\
MCA-M2 & $10(17 \%)$ \\
Basilar artery & $7(12 \%)$ \\
mTICl $\geq 2 b$ & $53(88 \%)$ \\
Prior allergic reactions & \\
Type of allergic reactions & \\
Nausea, vomiting & \\
Rash & $9(15 \%)$ \\
SOB, wheezing & $20(33 \%)$ \\
Anaphylaxis & $5(8 \%)$ \\
Other & $10(17 \%)$ \\
Not recorded & $5(8 \%)$ \\
\hline
\end{tabular}

Note:-TLKW indicates time last known well; SOB, shortness of breath; $\mathrm{mTICl}$, modified TICl.

Table 2: Precontrast exposure medication regimen and resultant reactions

\begin{tabular}{lc}
\hline \multicolumn{1}{c}{ Pre-Exposure Regimen } & No. (\%) \\
\hline Diphenhydramine and corticosteroid & $56(93 \%)$ \\
Corticosteroid only & $4(7 \%)$ \\
Medication specifics & $n(\%)$ \\
Diphenhydramine (median dose) & $56(93 \%), 50 \mathrm{mg}$ \\
Corticosteroids (median dose) & \\
Hydrocortisone & $48(80 \%), 200 \mathrm{mg}$ \\
Methylprednisolone & $11(18 \%), 100 \mathrm{mg}$ \\
Dexamethasone & $1(1.7 \%), 10 \mathrm{mg}$ \\
Postexposure reaction & \\
Nausea, vomiting & $0(0)$ \\
Rash & $0(0)$ \\
SOB, wheezing & $0(0)$ \\
Anaphylaxis & $0(0)$ \\
Other & $0(0)$ \\
\hline
\end{tabular}

Note:-SOB indicates shortness of breath.

Table 3: Impact of contrast allergy on time from hospital arrival to puncture

\begin{tabular}{ccc}
\hline Arrival to Puncture & $\begin{array}{c}\text { Mean } \\
\text { (SD) }\end{array}$ & $\begin{array}{c}\text { Median } \\
\text { (IQR) }\end{array}$ \\
\hline $\begin{array}{l}\text { Patients with prior documented contrast } \\
\text { allergy }(\mathrm{min})(n=60)\end{array}$ & $96(167)$ & $45(23-85)$ \\
All other patients $(\mathrm{min})(n=1403)^{\mathrm{a}}$ & $134(291)$ & $60(20-105)$ \\
\hline
\end{tabular}

${ }^{a}$ Excluding patients with incomplete data.

imaging and thereby pose a challenge to the neurointerventionalist advocating for workflow efficiency in obtaining care for the patient with a suspected LVO. For example, some radiology departments may require pretreatment medication before noninvasive vascular imaging (ie, CTA), resulting in delays to revascularization. At our institution, these delays ultimately resulted in the development of a protocol in which patients with contrast allergies bypass noninvasive vessel imaging and proceed directly to the neuroangiography suite.

In this report, we included those patients who underwent contrast exposure in the form of CTA at other institutions before transfer to our center for completion. However, in 43 patients older than 8 years of age who presented directly to our center and bypassed noninvasive imaging, proceeding with EVT as emergently as possible with premedication given en route resulted in no observable allergic reactions and numerically reduced the average time from hospital arrival to puncture (though this did not achieve significance).

Prior retrospective reports of patients undergoing percutaneous coronary interventions using ICM have demonstrated low rates of adverse reactions among patients with or without prior ICM allergy, leading some to advocate for reconsideration of the necessity of premedication with low-ionic ICM. ${ }^{11}$ Regardless, current recommendations from the American College of Radiology Manual on Contrast Media in 2020 are premedication regimens consisting of an antihistamine (eg, diphenhydramine) and a steroid (eg, hydrocortisone), with a minimum of 4 hours until contrast exposure for patients with documented ICM allergy. ${ }^{7}$ These guidelines were not designed for, nor are they tailored to, address the emergent nature of EVT. Our study may have relevance in other scenarios in which emergent contrast administration may be indicated in a timesensitive manner (eg, percutaneous coronary intervention, trauma, pulmonary embolism). The modified emergent premedication protocol used in our study could be considered in these other situations.

In 1986, Greenberger et $\mathrm{al}^{12}$ proposed a regimen of $200 \mathrm{mg}$ of intravenous hydrocortisone immediately, then every 4 hours until the procedure is completed, and $50 \mathrm{mg}$ of intravenous diphenhydramine 1 hour before emergency procedures. We have altered that protocol by delivering both classes of medications as soon as possible before needle puncture and by lowering exposure via bypassing noninvasive cerebrovascular imaging. Biologic effects of an intravenous hydrocortisone peak at 1-2 hours (half-life: 1.7 hours) ${ }^{13}$ and almost immediately after administration for intravenous diphenhydramine (half-life: 8.4 hours $) ;{ }^{14}$ despite these effects, there remains no definite proof that steroids or antihistamines given at the time of first contrast exposure, compared with several hours before a procedure, offer protection from an allergic reaction. ${ }^{7,15,16}$ However, because delaying EVT for LVO confers significant harm, the risk-to-benefit ratio favors intervention despite a potential allergic reaction. Thus, we believe that administering premedications en route to the neuroangiography suite, followed by close patient monitoring by trained staff for signs of allergy or reaction, is safe and practical. The data presented in this report represent a real-world, pragmatic, standardized approach to the care of a patient with an LVO and a known and/or documented contrast media allergy. While under ideal circumstances, patients with suspected LVO would be triaged with a noninvasive imaging technique, the unique challenge of balancing time to reperfusion with patient safety may justify a direct-to- 
angiography suite paradigm in this patient population with consideration of recommendations. If MR angiography without contrast can be performed without delay, this approach may be a reasonable alternative.

\section{Limitations}

This report has several limitations and must be interpreted with caution. First, this is a single-institution study of moderate size with no control cohort. Thus, this is not a valid comparison of one pretreatment paradigm versus any other. There were no patients with documented ICM allergies who did not receive premedication, and it is possible that the patients presented here would have the same outcomes had they not been administered premedication. Second, the cohort described here had documented allergies of varying severities and symptomatology and may not adequately sample patients with severe reactions. This analysis, predicated on documentation of allergic reactions to contrast media, may have excluded patients with an undocumented allergy. As with any procedure, the potential benefits of the procedure must be balanced against the risks for any individual patient. Third, delayed adverse reactions (most commonly urticaria $^{17}$ ) are rare but may develop from 30 to 60 minutes up to 1 week following exposure, with most occurring in the first 48 hours. $^{7,18}$ It is possible that minor delayed reactions beyond 24 hours would not be captured in this analysis. Fourth, determination of allergic reactions involving the airway is more difficult for the minority of patients who require intubation before undergoing EVT and may limit this analysis.

The strengths of this report are that the paradigm here consists of a standard regimen of widely available medications that are immediately accessible at most centers caring for these patients and results in minimal delays of patient care. The cohort reported here also includes patients with a variety of reactions and severities, therefore the results presented here are likely to have some degree of external validity.

\section{CONCLUSIONS}

In a single-institution cohort study of 60 consecutive patients with documented contrast allergies undergoing EVT for LVO with premedications administered en route to the neuroangiography suite, no patients experienced allergic symptoms. This pragmatic approach may be safe for patients who have documented contrast media allergies.

Disclosures: Shashvat M. Desai-RELATED: Comments: Award Number U10NS086489 (National Institutes of Health Stroke Trials Network, Regional Coordinating Center University of Pittsburgh).* Bradley A. Gross-UNRELATED: Consultancy: Medtronic and MicroVention. Brian T. Jankowitz-UNRELATED: Consultancy: Stryker, Medtronic. *Money paid to the institution.

\section{REFERENCES}

1. Sodagari F, Mozaffary A, Wood CG, 3rd, et al. Reactions to both nonionic iodinated and gadolinium-based contrast media: incidence and clinical characteristics. AJR Am J Roentgenol 2018;210:715-19 CrossRef Medline

2. Hunt $\mathrm{CH}$, Hartman RP, Hesley GK. Frequency and severity of adverse effects of iodinated and gadolinium contrast materials: retrospective review of $\mathbf{4 5 6 , 9 3 0}$ doses. AJR Am J Roentgenol 2009; 193:1124-27 CrossRef Medline

3. Deng F, Li MD, Wong A, et al. Quality of documentation of contrast agent allergies in electronic health records. J Am Coll Radiol 2019;16:1027-35 CrossRef Medline

4. Lasser EC, Lyon SG, Berry CC. Reports on contrast media reactions: analysis of data from reports to the U.S. Food and Drug Administration. Radiology 1997;203:605-10 CrossRef Medline

5. Brockow K. Contrast media hypersensitivity: scope of the problem. Toxicology 2005;209:189-92 CrossRef Medline

6. Kopp AF, Mortele KJ, Cho YD, et al. Prevalence of acute reactions to iopromide: postmarketing surveillance study of 74,717 patients. Acta Radiol 2008;49:902-11

7. American College of Radiology Committee on Drugs and Contrast Media. American College of Radiology Manual on Contrast Media. ACR;2020:22-26

8. Lang DM, Alpern MB, Visintainer PF, et al. Gender risk for anaphylactoid reaction to radiographic contrast media. J Allergy Clin Immunol 1995;95:813-17 CrossRef Medline

9. Davenport MS, Cohan RH, Caoili EM, et al. Repeat contrast medium reactions in premedicated patients: frequency and severity. Radiology 2009;253:372-79 CrossRef Medline

10. Mervak BM, Davenport MS, Ellis JH, et al. Rates of breakthrough reactions in inpatients at high risk receiving premedication before contrast-enhanced CT. AJR Am J Roentgenol 2015;205:7784 CrossRef

11. Topaz G, Karas A, Kassem N, et al. Iodinated contrast media allergy in patients hospitalized for investigation of chest pain. J Allergy Clin Immunol Pract 2018;6:2059-64 CrossRef Medline

12. Greenberger PA, Halwig JM, Patterson R, et al. Emergency administration of radiocontrast media in high-risk patients. J Allergy Clin Immunol 1986;77:630-34 CrossRef Medline

13. Derendorf H, Mollmann H, Barth J, et al. Pharmacokinetics and oral bioavailability of hydrocortisone. J Clin Pharmacol 1991;31: 473-76 CrossRef Medline

14. Blyden GT, Greenblatt DJ, Scavone JM, et al. Pharmacokinetics of diphenhydramine and a demethylated metabolite following intravenous and oral administration. J Clin Pharmacol 1986;26:529-33 CrossRef Medline

15. Lasser EC, Berry CC, Talner LB, et al. Pretreatment with corticosteroids to alleviate reactions to intravenous contrast material. $N \mathrm{Engl}$ J Med 1987;317:845-49 CrossRef Medline

16. Ring J, Rothenberger KH, Clauss W. Prevention of anaphylactoid reactions after radiographic contrast media infusion by combined histamine $\mathrm{H} 1$ - and $\mathrm{H} 2$-receptor antagonists: results of a prospective controlled trial. Int Arch Allergy Appl Immunol 1985;78:9-14 CrossRef Medline

17. Christiansen C, Pichler WJ, Skotland T. Delayed allergy-like reactions to $\mathrm{x}$-ray contrast media: mechanistic considerations. Eur Radiol 2000;10:1965-75 CrossRef Medline

18. Meth MJ, Maibach HI. Current understanding of contrast media reactions and implications for clinical management. Drug Saf 2006;29:133-41 CrossRef Medline 